\title{
Total Quality Management and Quality Circles in the Digital Lean Manufacturing World
}

\author{
David Romero ${ }^{1}$, Paolo Gaiardelli ${ }^{2}$, Daryl Powell ${ }^{3}$, Thorsten Wuest ${ }^{4}$, Matthias Thürer ${ }^{5}$ \\ ${ }^{1}$ Tecnológico de Monterrey, Mexico \\ david.romero.diaz@gmail.com \\ ${ }^{2}$ University of Bergamo, Italy \\ paolo.gaiardelli@unibg.it \\ ${ }^{3}$ Norwegian University of Science and Technology, Norway \\ daryl.j.powell@ntnu.no \\ ${ }^{4}$ West Virginia University, USA \\ thwuest@mail.wvu.edu \\ 5 Jinan University, China \\ matthiasthurer@workloadcontrol.com
}

\begin{abstract}
In this paper, the authors present practical insights in order to propose a Quality Management Framework for Digital Lean Manufacturing. The rationale behind this proposed framework is that the individual ISO 9000:2015 Quality Management pillars are rather different when it comes to their objectives and their tasks at hand when using Human capabilities and/or Digital technologies capabilities in a strategic way for (i) Quality Planning, (ii) Quality Control, (iii) Quality Assurance, and (iv) Quality Improvement in the context of the emerging paradigm of Digital Lean Manufacturing Systems.
\end{abstract}

Keywords: Digital Manufacturing, Smart Manufacturing, Lean Manufacturing, Digital Lean Manufacturing, Digital Lean Enterprise, Cyber-Physical Production Systems, Industry 4.0, Quality Control Circles, Total Quality Management.

\section{Introduction}

Today's manufacturing enterprises have to operate and thrive in highly competitive global environments where the highest quality, lowest cost, and shortest lead-time determine manufacturing competitiveness [1]. In this context, emerging paradigms such as Digital Lean Manufacturing (DLM) [2] offer an increase in the productivity and efficiency of manufacturing processes. In particular, DLM leverages the potential of cyber-physical quality management systems (i.e. tools, techniques, and practices) by increasing the stability and performance of such processes towards (near-)zero defect manufacturing [3].

According to Romero et al. [2], Digital Lean Manufacturing (DLM) "builds on new data acquisition, data integration, data processing and data visualization capabilities [4] to create different descriptive, predictive and prescriptive analytics applications [5] to detect, fix, predict and prevent unstable process parameters and/or avoid quality issues inside defined tolerance ranges that may lead to any type of waste within the cyber- and physical- worlds". Such capabilities contribute to support important feedback loops for quality improvement and the digitalization of quality management. 
In this paper, we propose a Quality Management Framework for Digital Lean Manufacturing based on the four ISO 9000:2015 pillars [6] of Quality Management: (i) Quality Planning, (ii) Quality Control, (iii) Quality Assurance, and (iv) Quality Improvement (see Fig. 1). Critical to this is the Lean principle of 'respect for people', which recognizes the unique contribution of human creativity, ingenuity and innovation (represented by the work of Quality Managers and their Quality Circles) to quality planning, quality control, and quality assurance tasks, and in particular to quality improvement duties, given the onset of digital technologies in combination with the new practices of quality management.

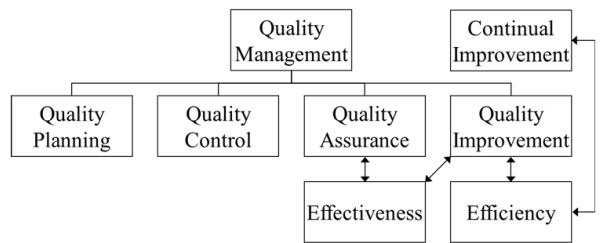

Fig. 1. A Quality Management Framework by ISO 9000:2015 [6]

The literature often argues that Quality Management $(Q M)$ practices in general, and the role of the Quality Manager in particular, will be substantially disrupted by the Fourth Industrial Revolution (a.k.a. The Industry 4.0 paradigm). Similar to the First

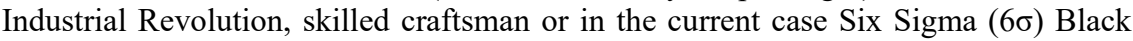
Belts will be substituted by 'technology' that can be operated by cheaper, less-skilled labour. In contrast, in this research, we seek to explore the still highly relevant roles of the Quality Manager, and his/her Quality Circles, in the QM practices of the future. In doing so, we distinguish between the four individual QM pillars of the ISO 9000: 2015 framework [6] in order to discuss and propose how a sensible, balanced, and valueadding Quality Management Framework could look like in an emerging "Digital Lean Manufacturing World".

\section{Towards a Quality Management Framework for DLM}

Total Quality Management (TQM) can be defined as a method, a continual process or a practice of detecting, reducing and eliminating errors in manufacturing processes in order to enhance their quality and productivity [7-9]. This can be done by streamlining workers' competencies through education and training programmes, statistical process controls, suppliers' controls and quality engineering controls (such as parameters and tolerances), so as to reduce manufacturing costs, and therefore waste, and increase customer satisfaction through continuous improvement [7-9]. Furthermore, Quality Control Circles $(Q C C)$, or simply Quality Circles, are small groups of workers that voluntarily and periodically meet to discuss production, quality, and related problems with the aim of collaboratively (cf. Nemawashi*) establishing productivity and quality goals and specifications as well as proposing improvements and corrective actions for those causes and issues constraining their achievement and compliance [10-12].

\footnotetext{
* In the Lean Manufacturing jargon, is the process of bringing problems and potential solutions to all those affected to gather their ideas and get agreement on a solution.
} 
From a DLM perspective, TQM and QCC have evolved over the decades. In the following $Q M$ continuum, this progress is loosely clustered building on the defining characteristics of the four Industrial Revolutions:

- QM 1.0 practices - based on human supervision of manufacturing processes and random physical inspection of products; and shop-floor meetings between workers for collaborative problem-solving.

- QM 2.0 practices - based on Statistical Process Control (SPC) [13] tools and techniques, using 'sampling-based measurements', to monitor, control, and improve a manufacturing process by early detection of its deterioration in order to safeguard product quality; and still face-to-face shop-floor meetings, with some 'phone-call' communications, between workers for collaborative problem-solving.

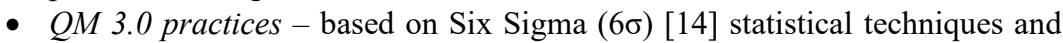
tools to identify and eliminate fluctuations in a manufacturing process in order to guarantee a uniform quality output; and first possibilities of virtual meetings between on-site and remote workers for collaborative problem-solving.

- QM 4.0 practices - based on (Big) Data Analytics and Machine Learning (ML) methods [15] capable of offering early alarms and fault diagnosis of problematic (manufacturing) processes, and therefore products, as well as optimisation parameters for optimized process settings. Moreover, advanced virtual meetings can now allow a true 'digital presence' of remote workers thanks to new interactive technologies such as mixed reality and haptics [16] for 'see-what-I-see', 'hear-what-I-hear' and even 'feel-what-I-feel' real-time collaborative problem-solving between remote and on-site workers.

In the context of $Q M 4.0$ practices, Romero et al. [2] have defined Digital Quality Management (DQM) as "the semi-automated or automated digital governance of cyberphysical production assets like smart products, smart operators and smart machines, aimed at offering real-time tracking and reporting of such intelligent assets performance towards compliance with predefined quality standards and proactively alerting in case of potential deviations from them to prevent quality problems (viz. defects) before they materialize". A similar concept to DQM is Virtual Quality Management (VQM) [17] [18], which in addition to production assets information, also considers environmental factors that can have a potential influence on manufacturing processes and on their quality outputs. It uses modelling and simulation techniques in order to try, test and optimize virtual process chains prior to setting-up the real manufacturing processes in the factory [18]. Hence, emerging Digital Lean Cyber-Physical Production Systems (CPPSs) [2] [19], characterized by their real-time feedback loops, offer a huge potential for DLM and VQM implementations.

The following sub-sections (see 2.1 to 2.4) describe an enhanced QM framework with Lean principles and Digital technologies (e.g. Industrial Internet of Things (IIoT), (Big) Data Analytics, Cyber-Physical Systems (CPSs), Machine Learning (ML), Edge Computing) in order to introduce a Quality Management Framework for DLM.

The rationale behind this proposed framework is that the individual QM pillars are rather different when it comes to their objectives and their tasks at hand when using Human capabilities and/or Digital technologies capabilities in a strategic way. 


\subsection{Quality Planning for Digital Lean Manufacturing}

Based on ISO 9000:2015 [6], the first pillar of QM is 'Quality Planning'. This activity is focused on "setting quality objectives and specifying necessary operational processes and resources to fulfil these quality objectives". It requires the agreement of relevant stakeholders, including internal and external customers, to ensure that their expectations for quality requirements and legal compliance are correctly identified. In this context, even though digital technologies (e.g. advanced modelling, simulation, and (big) data analytics tools [20]) have made a lot of progress as 'supporting tools' for quality planning, Quality Managers, and their Quality Circles, still play an important role. This vital role includes but is not limited to, their human touch (i.e. sensitivity) when it comes to identifying and understanding customer requirements (i.e. the Voice of the Customer [21]), setting quality criteria, and defining quality standards. Since expressed quality specifications will never cover exactly all the quality aspects expected by the end-user as highlighted by [22], there is a distinction between 'relevant quality', also known as service quality, covered by crucial technical quality specifications, and 'cosmetic quality', synonym of specified quality, covered by crucial customer quality specifications. Moreover, if we use only technology for quality planning, we will limit our human capabilities to conduct this important QM activity manually, and we will not be able to properly teach the workers about quality.

\subsection{Quality Control for Digital Lean Manufacturing}

ISO 9000:2015's [6] second pillar of QM is 'Quality Control'. This activity is focused on "fulfilling quality requirements". It is responsible for determining whether quality acceptance criteria have, or have not, been met according to quality specifications, using different inspection and testing techniques. Quality Control Systems benefit from new, and improved digital technologies, such as (big) data-driven industrial process monitoring, fault-detection and diagnosis technologies [23] [24], machine (computer) vision inspection systems [25], and edge computing processes [26]. Supervised, semisupervised and un-supervised machine learning algorithms [27] are capable to identify quality deviations early in the process, potentially enabling rework of problematic parts and thus reducing scrap (waste). While this reduces the direct involvement of (human) Quality Experts, such as replacing human operated visual inspection stations, there are other areas that emerge that may need to be staffed with the humans' unique skills. For example, insightful management of 'data quality' is still an issue that causes problems with automated, (big) data-driven industrial process monitoring, fault-detection and diagnosis systems [23], and Quality Managers, and their Quality Circles are key for addressing these data quality challenges today.

\subsection{Quality Assurance for Digital Lean Manufacturing}

ISO 9000:2015's [6] third pillar of QM is 'Quality assurance'. This activity is focused on "providing confidence that quality requirements will be fulfilled according to planned activities and results". It validates the consistent use of quality control procedures and standards and ensures that human and artificial quality control systems have the correct capabilities to fulfil their responsibilities. Quality Assurance builds on the insights from the collected information and data of production operations. Here a combined approach 
between qualified Quality Managers and (big) data analytics are often the method of choice when it comes to dealing with the production complexity. (Big) data-driven approaches provide the input, within a variety of degrees, yet the Quality Managers are the ones putting the data into context and interpreting it, and drawing the conclusions that lead to quality improvement actions. Thus, at all times, the Quality Experts need to have a solid understanding of what the data means (i.e. interpreting correctly the data to infer the right information from it in order to properly answer questions and/or take actions), and of what the (big) data-driven systems actually do and how their results are developed (i.e. explain the functioning of the data analytical models). Black-box systems are powerful, yet problematic in a "Digital Lean Manufacturing Systems Environment" where workers are supposed to constantly pursue continuous learning and productprocesses quality improvement(s). Therefore, employees commitment to continuous learning will remain a critical factor for ensuring quality. This is because the workers will continue to control operations or rather take the decisions on quality. Consequently, Quality Circles are key to deal with (production) uncertainty and to create a sustainable quality assurance strategy, since manufacturing enterprises cannot only rely on nonexplainable 'artificial intelligence(s)' [see 28] and/or external experts when it comes to the know-how to perfect (i.e. to make lean and digitalize) their manufacturing processes for operational excellence and high-quality standards. Quality Managers, and their Quality Circles, should always be part of the journey of perfecting any manual or semiautomated process before it becomes to be fully automated in order to avoid the "blackbox problem", since anyone could buy quality control and quality assurance systems, but the knowledge of how to use them strategically and effectively must be acquired and developed by the employees.

\subsection{Quality Improvement for Digital Lean Manufacturing}

ISO 9000:2015's [6] fourth pillar of QM is 'Quality Improvement'. This activity is focused on "increasing (recurrently) the ability to fulfil quality requirements as they evolve". It drives the quality continual improvement by using the information provided by the quality assurance and the quality control activities to improve effectiveness and efficiency. Moreover, Quality Improvement can be generally divided into two types: (i) incremental quality improvements (cf. Kaizen) - driven by (real-time) optimization techniques of processes [29], and supported by humans, and (ii) breakthrough quality improvements (cf. Kaikaku) - driven by human creativity, ingenuity and innovation, and supported by digital technologies. In this context, even though 'technology' has gone far with advanced modelling, simulation, and optimization techniques as well as with Machine Learning (ML) algorithms, machines may learn something that the ML algorithms' developers did not initially program or set, consequently, machine-learned results should be validated by humans, even if unexpected. Therefore, it is important to not underrate the humans' creativity and ingenuity. Moreover, without these human capabilities, a 'smart factory' may remain stuck at the same stage of development as the optimization techniques reach their limits and improvements become marginal. Therefore, Quality Managers and their Quality Circles are key for true continuous quality improvements and for the development of quality competitive advantages. Hence, we need to continue investing in and empowering our employees, our Quality Experts and their ideas and skills, even when digital technologies seem to be very progressive. 


\section{Discussion \& Reflections}

The aim of this paper is to explore the still highly relevant roles of the Quality Manager, and his/her Quality Circles, in the QM practices of the future, framed in light of the four individual QM pillars of the ISO 9000:2015 framework [6]. We discuss and propose how a sensible, balanced, and value-adding Quality Management Framework could look like in an emerging "Digital Lean Manufacturing World" [2]. In general, although the pillars of Quality Control and Quality Assurance indeed have the potential to become highly automated in light of (smart) digital technologies [30] [31], Quality Planning and Quality Improvement pillars may potentially require more human creativity, ingenuity and innovation than ever before, combining traditional skills of continuous improvement and QM techniques with the (new) smart capabilities presented by digital technologies.

Fig. 2 represents our proposed Quality Management Framework for DLM, built on the four pillars of QM [6], where we highlight the Human (H) or Automation (A) heavy nature of the pillars in the QM practices of the future, given the onset of (smart) digital technologies and the Industry 4.0 paradigm.

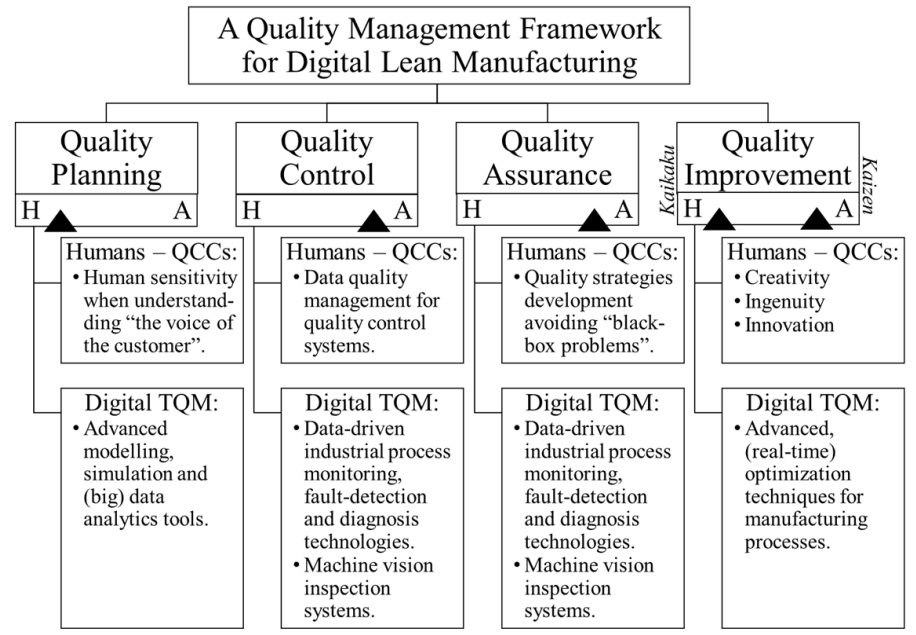

Fig. 2. Towards a Quality Management Framework for Digital Lean Manufacturing

Quality Planning - is expected to have a continued reliance upon human creativity, ingenuity and innovation regardless of the degree of (smart) digital technologies used. Quality planning is a customer-sensitive task, and even though humans may be able to benefit from greater use of advanced modelling, simulation, and (big) data analytics tools, the quality planning task will remain human-intensive in future QM practices. On the other hand, Quality Control - will almost certainly become automation-heavy. The onset of (smart) digital technologies for process monitoring, process control, faultdetection and fault-diagnosis in addition to low-cost visual inspection systems provide manufacturers with incentives to automate quality control tasks. This also stands true 
for Quality Assurance tasks - drawing on the same technologies as quality control. However, quality assurance will continue to draw on the creativity and ingenuity of the human, where Quality Managers, and their Quality Circles, define and execute strategies for effective quality assurance. Finally, the role of the Quality Manager, and his/her Quality Circles, is set to become even more essential in Quality Improvement - given the current shift towards the Industry 4.0 paradigm, where time saved by automating the quality control and quality assurance tasks can be much better spent on quality improvement, enabling manufacturing enterprises to successfully operate and thrive in highly competitive environments where the highest quality, lowest cost, and shortest lead-time determine an organisation's ultimate manufacturing competitiveness. In Quality Improvement, small, continuous process improvements can be automated to a higher degree through smart process optimization technology. However, larger improvement possibilities, radical innovations, will be realized to a greater extent through harnessing human creativity, ingenuity and innovation applied to problemsolving in DLM environments.

\section{Conclusions}

Industry 4.0 presents manufacturing enterprises with a plethora of new technologies [31] that promise greater competitiveness through higher quality, lower cost, and shorter lead-times. However, following the true spirit of Lean Manufacturing, firms should not neglect the power of the 'respect-for-people' principle, even though the promise of automation is extremely attractive.

In this paper, we highlight several avenues in which the QM practices of the future can benefit from new (smart) digital technologies when combined with greater levels of human creativity, ingenuity and innovation - utilizing their unique capability for collaborative problem-solving. At this stage, the analysis relied mainly on insights from literature as well as from scholarly and industry experts and is more explorative and theoretical in nature. This limitation should be addressed through rigorous empirically grounded work, assessing the elements of this QM Framework for DLM based on data collected from QM departments of various industries.

\section{References}

1. Singh, M., Khan, I.A., Grover, S.: Tools and Techniques for Quality Management in Manufacturing Industries. National Conference on Trends and Advances in Mechanical Engineering, pp. 853-859 (2012)

2. Romero, D., Gaiardelli, P., Powell, D., Wuest, T., Thürer, M.: Digital Lean Cyber-Physical Production Systems: The Emergence of Digital Lean Manufacturing and the Significance of Digital Waste. Part I, IFIP AICT 535:11-20 (2018)

3. Eleftheriadis, R., Myklebust, O.: A Quality Pathway to Digitalization in Manufacturing thru Zero Defect Manufacturing Practices. International Workshop on Advanced Manufacturing and Automation, pp. 187-191 (2016)

4. Kusiak, A.: Smart Manufacturing Must Embrace Big Data. Nature, 544(7648):23-25 (2017)

5. Wuest, T., Weimer, D., Irgens, C., Thoben, K.-L.: Machine Learning in Manufacturing: Advances, Challenges, and Applications. J. Production \& Mfg. Research, 4(1):23-45 (2016)

6. ISO 9000: Quality Management Systems - Fundamentals and Vocabulary (2015)

7. Crosby, P.: Quality is Free. New York, McGraw-Hill (1969) 
8. Juran, J.M.: Quality Control Handbook. New York, McGraw-Hill, pp. 5-12 (1979)

9. Deming, E.: Out of the Crisis: Quality, Productivity and Competitive Position. Cambridge, Massachusetts, MIT Press (1982)

10. Ishikawa, K.: What is Total Quality Control? The Japanese Way. Prentice Hall (1985)

11. Hill, S.: From Quality Circles to Total Quality Management. Making Quality Critical: New Perspectives on Organizational Change. Routledge: London, pp. 33-53 (1995)

12. Hill, F.M.: Organizational Learning for TQM through Quality Circles. The TQM Magazine, 8(6):53-57 (1996)

13. Montgomery, D.: Introduction to Statistical Quality Control. NY: John Wiley \& Sons (2005)

14. Antony, J. Banuelas, R., Kumar, A.: World Class Applications of Six Sigma: Real World Examples of Success. $1^{\text {st }}$. Edition, Oxford Elsevier, Butterworth-Heinemann (2006)

15. Li, X. et al.: Deep-Level Quality Management based on Big Data Analytics with Case Study. Chinese Automation Congress, IEEE, pp. 4921-4926 (2017)

16. Perera, C., Liu, C.H., Jayawardena, S.: The Emerging Internet of Things Marketplace from an Industrial Perspective: A Survey. IEEE Transactions on Emerging Topics in Computing, 3(4):585-598 (2015)

17. Bodi, S., Popescu, S., Drageanu, C., Popescu, D.: Virtual Quality Management Elements in Optimized New Product Development using Genetic Algorithms. MakeLearn and TIIM Joint International Conference Proceedings, pp. 633-642 (2015)

18. Bookjans, M., Weckenmann, A.: Virtual Quality Management - Validation of Measurement Systems by the Use of Simulation Technologies. Physics Procedia, 5:745-752 (2010)

19. Powell, D., Romero, D., Gaiardelli, P., Cimini, C., Cavalieri, S.: Towards Digital Lean Cyber-Physical Production Systems: Industry 4.0 Technologies as Enablers of Leaner Production. Part II, IFIP AICT 535:353-362 (2018)

20. Shao, G., Shin, S.-J., Jain, S.: Data Analytics using Simulation for Smart Manufacturing. Proceedings of the Winter Simulation Conference (2014)

21. Johnson, G.: Your Customers Still Want to Talk to A Human Being. HBR (2017)

22. Gunsteren, L.A.: Quality in Design and Execution of Engineering Practice. Vol. 7, IOS Press (2013)

23. Qin, S.J.: Survey on Data-driven Industrial Processes Monitoring and Diagnosis. Annual Reviews in Control, 36(2), pp. 220-234 (2012)

24. He, P., Wang, J.: Statistical Process Monitoring as a Big Data Analytics Tool for Smart Manufacturing. Journal of Process Control, Vol. 67, pp. 35-43 (2018)

25. Beyerer, J., Puente León, F., Frese, C.: Machine Vision - Automated Visual Inspection: Theory, Practice and Applications. Springer-Verlag Berlin Heidelberg (2016)

26. Chen, B., Wan, J., Celesti, A., Li, D., Abbas, H., Zhang, Q.: Edge Computing in IoT-Based Manufacturing. IEEE Communications Magazine, 56(9):103-109 (2018)

27. Tsironis, L., Bilalis, N., Moustakis, V.: Using Machine Learning to Support Quality Management: Framework and Experimental Investigation. The TQM Magazine, 17(3):237248 (2005)

28. Gunning, D.: Explainable Artificial Intelligence (XAI). DARPA-BAA-16-53 (2016)

29. Venkata, R.R.: Advanced Modeling and Optimization of Manufacturing Processes International Research and Development. Springer-Verlag London (2011)

30. Illés, B., Tamás, P., Dobos, P., Skapinyecz, R.: New Challenges for Quality Assurance of Manufacturing Processes in Industry 4.0. Solid State Phenomena, 261:481-486 (2017)

31. Mittal, S., Kahn, M., Romero, D., Wuest, T.: Smart Manufacturing: Characteristics, Technologies and Enabling Factors. Part B: Journal of Engineering Manufacture, pp. 1-20, DOI 10.1177/0954405417736547 (2017) 